\title{
Bio safety Knowledge, Waste Disposal Practices and Identification of Parasites in Biomedical Wastes from Healthcare Establishments in Lagos and Abuja, Nigeria
}

\author{
Abiodun A Denloye ${ }^{* 1}$, Oladele T Ojuromi ${ }^{1}$, Kingsley Ochei ${ }^{2}$ and Elizabeth A Ade-Serrano ${ }^{1}$ \\ ${ }^{1}$ Department of Zoology and Environmental Biology, Lagos State University Ojo, Nigeria \\ ${ }^{2}$ Family Health International, Abuja, Nigeria
}

Received: June 11, 2018; Published: June 19, 2018

*Corresponding author: Abiodun A Denloye, Department of Zoology and Environmental Biology, Lagos State University Ojo, Lagos, Nigeria, West Africa

\begin{abstract}
In Nigeria, the level of bio safety awareness has not been established especially among workers in Health Care Establishments (HCEs), neither have pathogenic organisms in wastes from HCEs been identified. Studies were therefore carried out to highlight the level of bio safety awareness and the biomedical waste disposal practices in selected HCEs in Abuja and Lagos respectively as well as identify parasites from HCE wastes from Lagos prior to final disposal. A total of 200 questionnaires were administered to HCE workers in Abuja and Lagos respectively for information on their knowledge of bio safety and how their wastes are disposed of. Also, samples of urine (40) and stools (50) from 90 HCEs in Lagos were examined for presence of parasites using standard parasitological methods comprising of direct wet mount and formol ether concentration techniques. Both urine and stool were examined with 5\% lugol iodine and kinyoun carbol fuschin stain. Result shows that HCE workers had knowledge of the basics of bio safety but those in Lagos understood more than the ones in Abuja. Trichomonas vaginalis was identified in one of the urine samples. Female stool (22\%) had the highest prevalence of intestinal parasites relative to males (4\%). Entamoeba histolytica (6\%), Ascaris lumbricoides (6\%) and Cryptosporidium spp (6\%) were prevalent in the stool samples. These results underscore the need for more enlightenment and education for HCE workers in respect of bio safety. It further shows that the HCEs in Lagos dispose of their biomedical wastes improperly, which can lead to further transmission of pathogenic organisms.
\end{abstract}

Keywords: HCEs: Healthcare establishments, CDC: Centers for Disease Control, HCEs: Health Care Establishments, LAWMA: Lagos State, the Waste Management Authority

Abbreviations: Bio Safety Knowledge; Health Care Establishments; Biomedical Waste Disposal; Parasites; Parasitological Methods; Centers For Disease Control; Care Establishments; Cryptosporidium Species; Capital Territory; Entamoeba Histolytica; Gardia Lamblia; Non-Existent Segregation; Ascaris Lumbricoides; Trichuris Trichiura

\section{Introduction and Background to Study}

Healthcare establishments (HCEs) analyze, generate, handle, treat and dispose of biomedical wastes of various types including pathogenic microorganisms. Workers in these HCEs including sicentists, researchers, students, technologists and attendants are often exposed to infectious agents, processes and equipment. Also the products of technological processes which include foods and genetically modifed organisms (including plants) may lead to hazardous consequencies to which man is exposed. The principles and practices of bio safety are therefore of extreme importance. Bio safety is the avoidance of risk to human health and safety and conservation of the environment, as a result of the use of research and commerce of infectious and genetically modified organisms [1]. Also, the Centers for Disease Control (CDC) define bio safety as the discipline addressing the safe handling and containment of infectious microorganisms and hazardous biological materials [2]. Owing to its impact on human and environmental health, the principles and practices of bio safety are very relevant in Health Care Establishments (HCEs) which, because of their activities receive, handle, treat and dispose of various pathogenic organisms. These activities generate wastes of various kinds including sharps, swabs, syringes, corrugated packaging materials, paper, dyes, other chemcials and so on. Furthermore, HCE wastes also include Infectious wastes like human and animal specimen culture from medical and pathological labs, waste contaminated with blood and by-products , cultures and stocks of biohazard agents; waste from patients in isolated wards, discarded diagnostic samples containing blood and body fluid, dis- 
carded live and attenuated vaccine culture devices used to transfer inoculate and mix culture. Contaminated materials such as swabs and bandages as well as contaminated devices such as disposable medical devices. Also, they may include pathological wastes such as recognizable body and animal parts, tissue fluids or carcasses suspected of being infected with infectious agents known having zoonotic potentials. Wastes generated from pharmaceuticals such as expired drugs, unused and contaminated drugs, vaccines, sera, geotaxis and heavy metals can also have profound effect on human health and the environment. It is estimated that HCE wastes are composed mainly of general wastes $(80 \%)$ and hazardous materials $(20 \%)$ which may be infectious, toxic or radioactive [3]. The infectious component of HCE waste contains potentially harmful microorganisms capable of infecting hospital patients, health care workers, pets, livestock and the general public.

The foregoing underscores the need for bio safety knowledge and good management practice for biomedical wastes. There is a dearth of studies in this respect except for the report of Stephen, Elijah [4] on health care Waste management in a teaching hospital in Edo State, Nigeria. Also, the level of bio safety awareness among workers in HCEs in Nigeria is not known, neither have the pathogenic organisms in their wastes identified. The present study highlights the level of bio safety awareness and the biomedical waste disposal practices in selected HCEs in Abuja and Lagos respectively and also identifies parasites from HCE wastes in Lagos prior to final disposal. The study was carried out to evaluate the level of bio safety awareness in selected HCEs, document the medical waste disposal practices in Lagos and Abuja respectively and identify parasitic organisms in biomedical wastes from HCEs in Lagos prior to final disposal.

\section{Materials and Methods}

\section{a) Location of HCEs and Administration of questionnaires}

Two locations were selected as study areas namely Lagos and Abuja due to their cosmopolitan nature as well as their relative importance to Nigeria politically and economically. Abuja is the seat of government at the federal level being the administrative capital of Nigeria, and Lagos is the commercial capital serving as host to more than 2000 industries and being the former ad- ministrative capital of Nigeria. Structured questionnaires were administered in selected HCEs in Lagos and Abuja respectively with the aim of extracting information on the level of bio safety knowledge among various categories of HCE workers in the two centre's and how their biomedical wastes are disposed of. A total of 200 questionnaires were administered in Lagos (100) and Abuja (100) with a recovery rate of $82 \%$ and $79 \%$ for each study area respectively.

\section{b) Collection and Analyses of Samples}

Urine and stool waste samples were collected respectively from holding points in selected HCEs located in Lagos. The samples comprised of $56 \%$ stool and $44 \%$ urine samples. Fresh stool and urine samples were collected using clean universal sample bottles with tight fitting lids. After collection, the samples were transported in ice-pack to the laboratory for examination. Urine samples were transported to the laboratory and the samples maintained in a deep freezer at temperature of about -20oC before examination. Each sample bottle was labeled for type, age, sex and date of collection. The sample tube was half-filled and saline solution added. Centrifugation was carried out at 2000rpm for 2 mins. The supernatant was decanted and sediment was examined with $5 \%$ lugol iodine not to miss the trophozoites of Entamoeba and Giardia. Stool samples were visually examined under the microscope to note the consistency, presence of abnormal features, whether they are watery, bloody or mucous. Stool samples were later processed through formol ether concentration techniques as described by Garcia [5] the observations from formol ether concentration technique were used for analyses.

\section{Results}

a) Knowledge of bio safety among HCE workers in Lagos and Abuja: Analysis of questionnaires showed that majority of respondents in either Lagos or Abuja had received training while some HCE workers had no knowledge of bio safety. The results also showed that more workers in Lagos had received formal training in bio safety and there were more HCE workers in Abuja that understood the concept of bio safety than the workers from Lagos HCEs (Figures 1a and 1b).

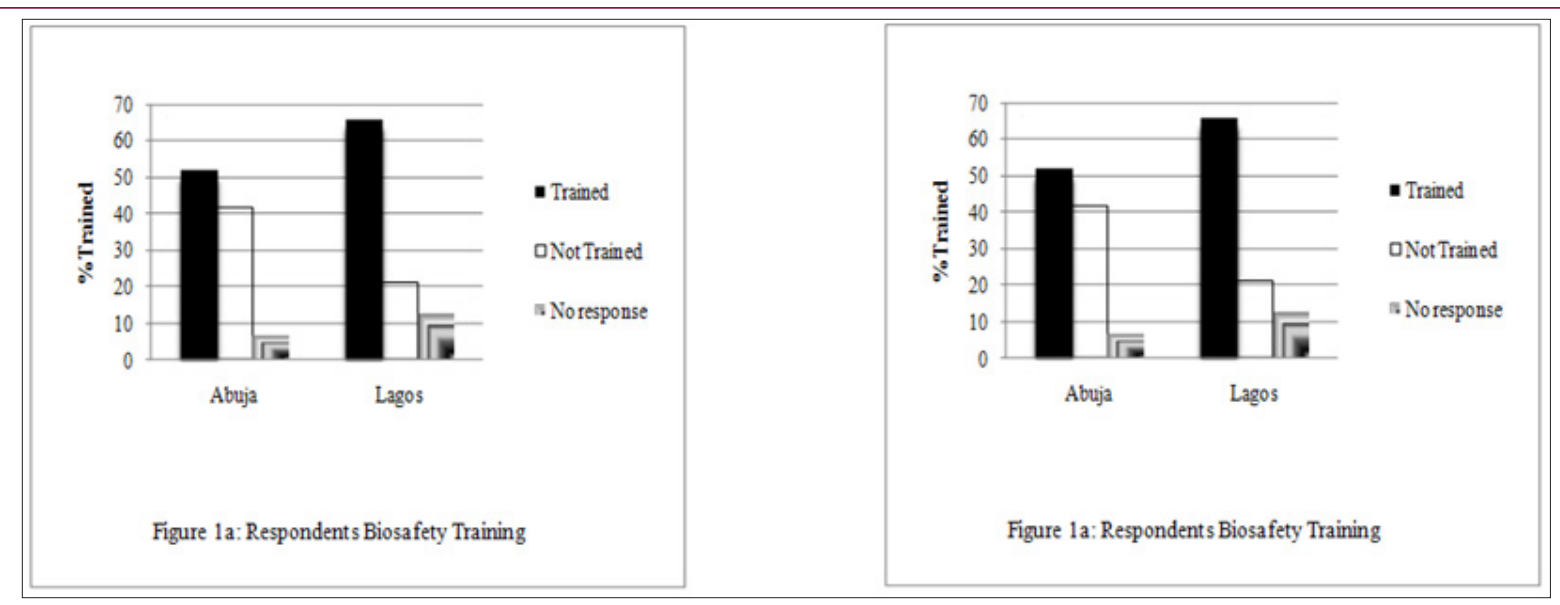

Figure 1: Level of bio safety training and understanding in Lagos and Abuja. 
b) Biomedical Waste Characterization by HCEs in Lagos and Abuja: The biomedical wastes from HCEs in the two study locations were identified as urine, blood, stools, sputum, semen/ vaginal discharge, and skin snip. Some of the wastes from Abu- ja could not be identified and characterized and were consequently group as others. There were more of all identifiable biomedical wastes in Lagos HCEs than those from Abuja (Figure 2).

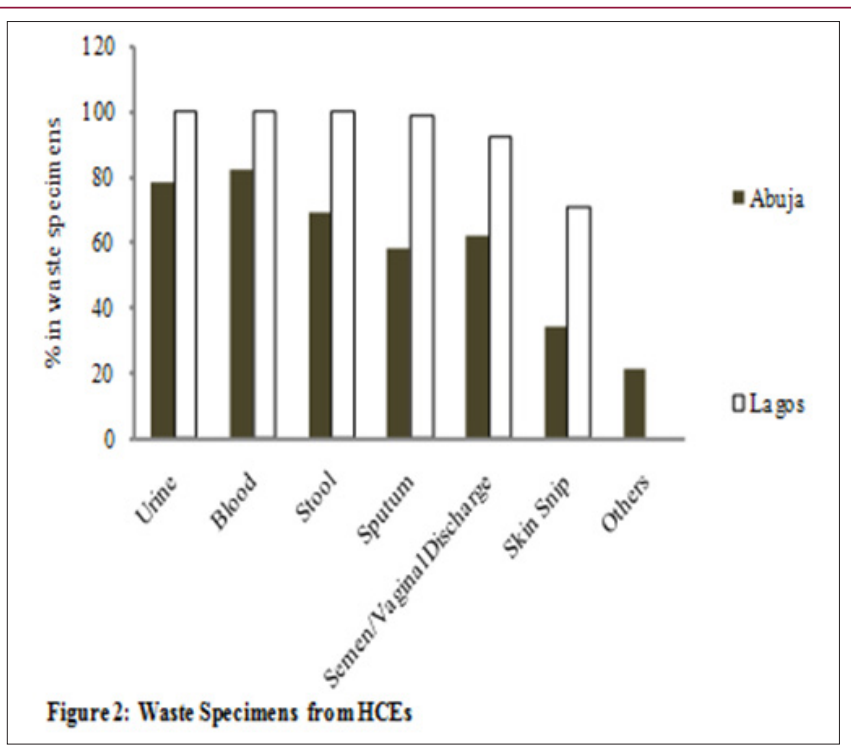

Figure 2: Waste Specimens from HCEs

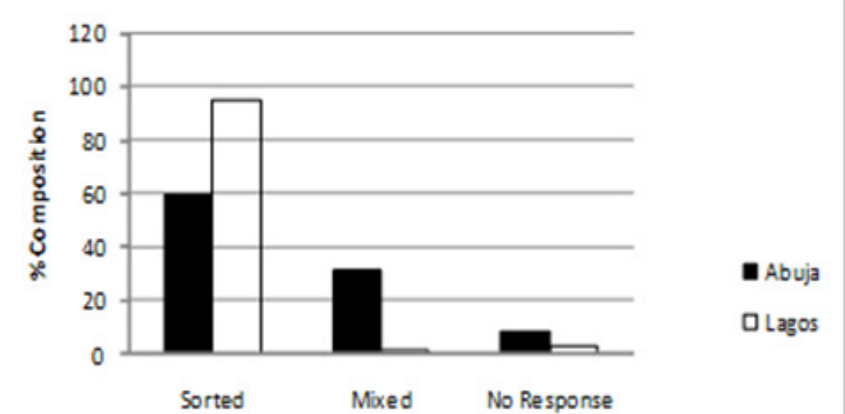

Figure 3a: Sorting of solid wastes by HCEs in Storage

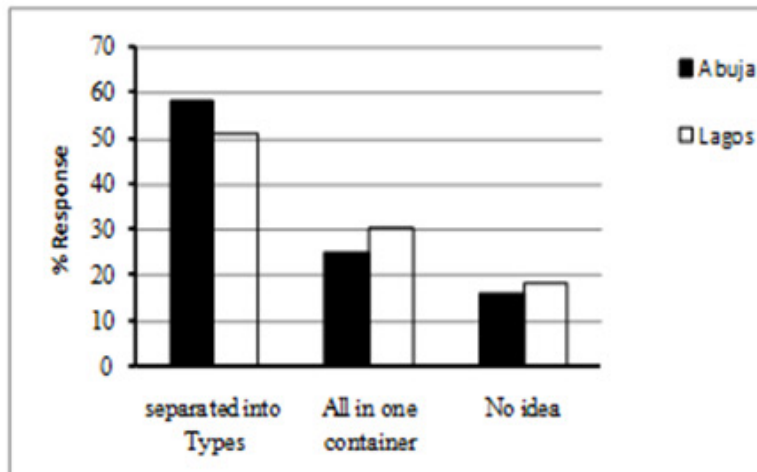

Table 3b: Separation of Liquid wastes by HCEs

Figure 3: Sorting of solid and liquid wastes from HCEs in Lagos and Abuja.
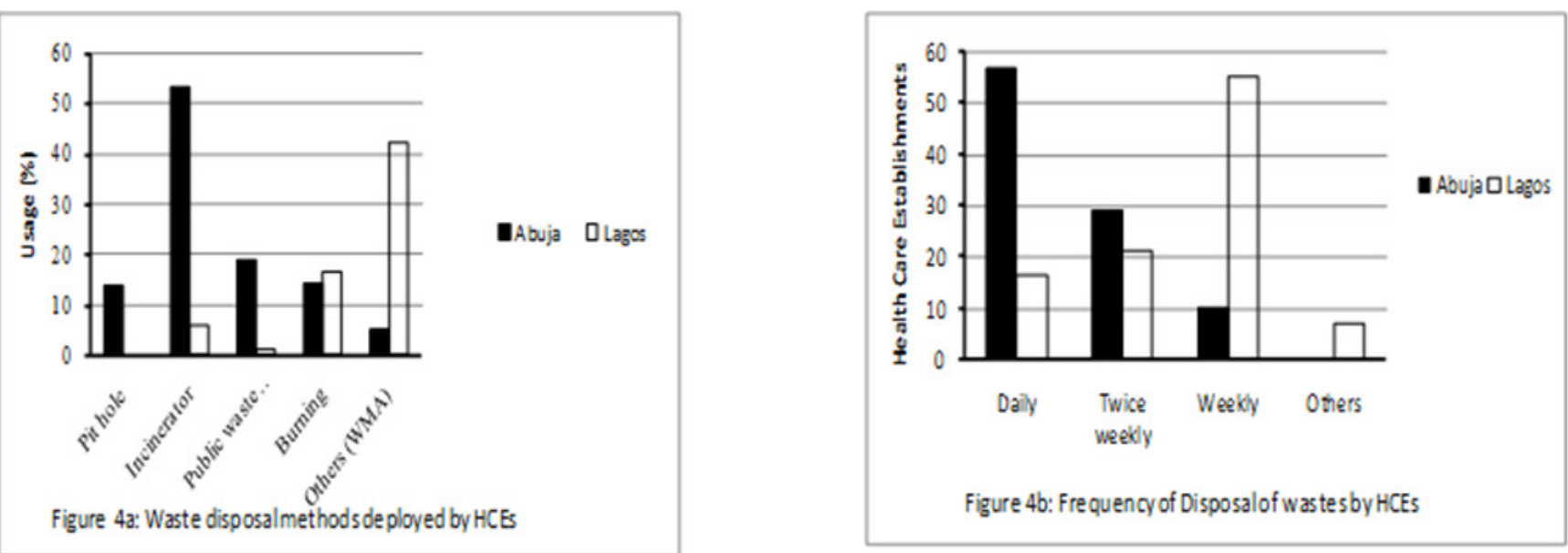

Figure 4: Methods and frequency of biomedical waste disposal in Lagos and Abuja 
c) Biomedical waste disposal practices of HCEs in Lagos and Abuja: In the two study locations, the wastes were sorted prior to final disposal. More sorting was recorded in Lagos relative to Abuja HCEs. All solid wastes were sorted by respondents from Lagos while $60 \%$ or less sorted their wastes in Abuja (Figure 3a). More of the liquid wastes were separated into types in Lagos than in Abuja (Figure 3b). Also, HCEs in Abuja dispose of their biomedical wastes by pit hole, incineration, public waste disposal point, and Waste Management Authority while none of the HCEs in Lagos use pit method. In addition, most of the Abuja HCEs use incinerator while most of those in Lagos dispose of their wastes through services of the Waste Management $\mathrm{Au}$ - thority (Figure 4a). Most HCEs in Abuja dispose of their wastes more frequently (daily) than the ones in Lagos (weekly) (Figure 4b).

d) Parasitic Organisms in Biomedical Wastes from Lagos HCEs: One of the urine samples tested positive for Trichomonas species. Most of the stool samples (72\%) had no parasite. Four protozoans (Cryptosporidium species, Entamoeba histolytica, Gardia lamblia and Trichomonas vaginalis), one flat worm (Schistosoma mansoni) and two nematodes (Trichuris trichiura and Ascaris lumbricoides) were identified in the stool samples (Figure 5). Most of the stool samples from which parasites were identified were collected from female patients (Figure 6).

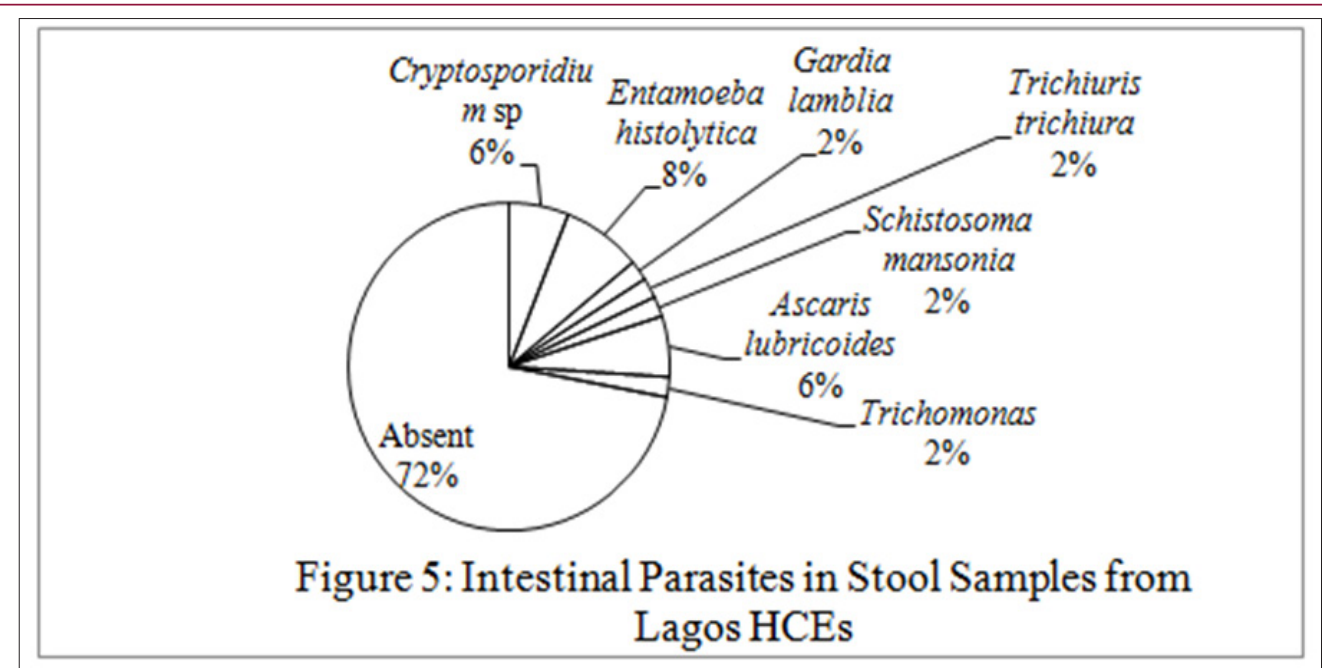

Figure 5: Intestinal parasites in Stool Samples from Lagos HCEs.

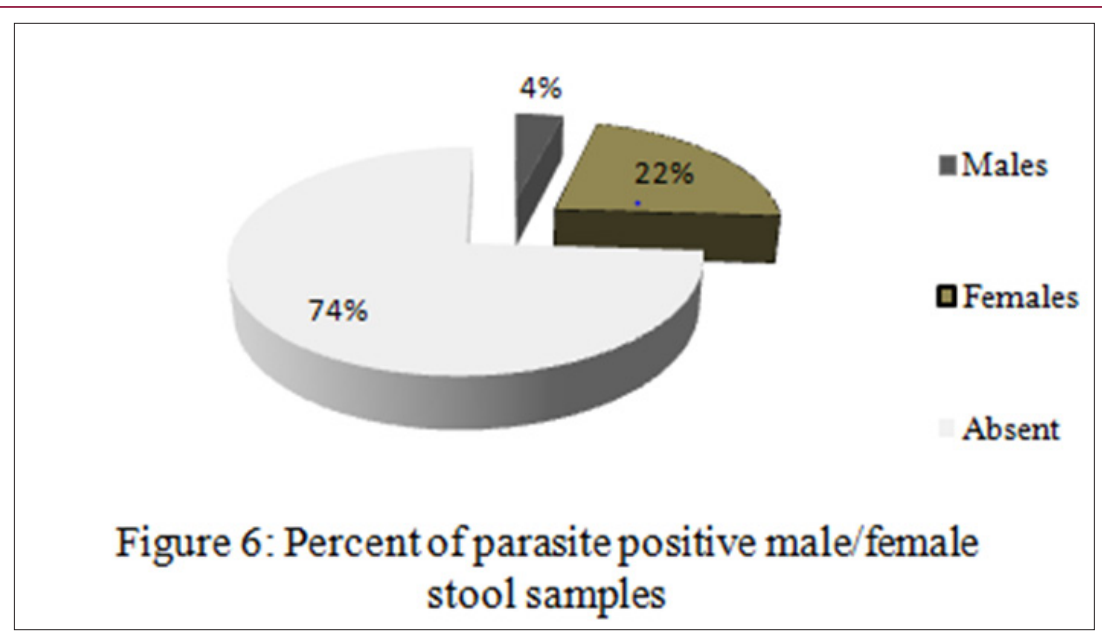

Figure 6: Percent of parasite positive male/female stool samples

\section{Discussion}

This study reveals the knowledge and practice of bio safety among workers in Health Care Establishments (HCES), biomedical waste disposal and the occurrence of pathogenic microbes in the wastes from HCEs in two Nigerian cities prior to final disposal. The results of this study show that more HCE workers in Lagos understood the basic principles of bio safety more than those in Abuja. This may be because Lagos State has a better organized waste disposal system relative to the Abuja. In Lagos State, the Waste Man- agement Authority (LAWMA) is actively involved in the disposal of biomedical wastes using LAWMA-branded medical waste truck. The LAWMA also give out color-coded boxes for waste segregation prior to final disposal. In Abuja, this active involvement of a Waste Management Authority in medical waste disposal is lacking. The foregoing non-committal situation with respect to medical waste disposal in Abuja by the waste board typifies the lack of commitment by management of waste disposal boards as shown by Stephen and Elijah [4]. 
The lack of management commitment, poor waste handling practices, inadequate training of Health Care Workers (HCWs), non-existent segregation of HCW and risky disposal were the bane of a teaching hospital studied in Nigeria [4]. In alluding to the poor handling of biomedical wastes, Ohakwe [6] reported that HCEs in Enugu State, eastern Nigeria, store infectious wastes in plastic buckets, disposable bags, waste bins or rubber buckets without a clear indication of its content and that chemicals are poorly disposed of in sink without any form of treatment, allowing the untreated waste chemicals to run into open or underground pit. Such disposed waste chemicals pose serious environmental hazard in the near and distant future.

Responses to questionnaires in this study also showed that HCE wastes in both Lagos (18.82 \%) and Abuja (50.63\%) were not treated prior to final disposal. The disparity in these figures is likely to be a result of the understanding of the principles of bio safety and their putting them into practice by Lagos HCE workers relative to those in Abuja as earlier stated. It however shows that HCEs that failed to treat their wastes prior to final disposal do so in conformity with the general practice in Nigerian HCEs as reported by Stephen and Elijah [4]. It is established that the common practice in Nigeria is that the wastes are collected at a central open dumpsite and burnt periodically. Occasionally, the wastes are buried by covering with a heap of soil without any prior treatment. Furthermore, human body parts such as placenta and amputated limbs are either disposed of with the general HCE wastes or given to the patients or their relatives to dispose of in their own way [5].

Some intestinal parasites including diarhoea-causing protozoans (Entamoeba histolytica, Gardia lamblia and Cryptosporidium sp), Helminths (Ascaris lumbricoides, Trichuris trichiura) and Schistosome (Schistosoma mansoni) were identified in samples disposed of by HCEs [6]. The presence of these parasites in wastes gives strong grounds to conclude that the wastes did not receive prior treatment. The presence of parasites shows that the handling of wastes from the HCEs is unsustainable and cannot be relied upon for the protection of human health and environmental integrity because healthy humans and animals in proximity with the HCEs in Lagos may get infected with these parasites $[7,8]$. It is unclear how long the parasites identified in these wastes would survive and cause infection, some enteric parasites such as E. histolytica cysts have been reported to survive for up to 30 days [9]. Also, Giardia cysts could survive for up to 24 days at sub room temperature [10] while A. lumbricoides can stay alive for up to 20 years or more [11]. It is interesting to note that Cryptosporidium oocysts have been found to survive and retain their infectivity for several months in both salt, fresh water and chlorinated water [12]. The period of temporary stay at the HCE vicinity is therefore sufficient for the parasites to still be infective. The diseases caused by the presence of the identified parasites would therefore continue to recur and spread in the environment.

\section{Conclusion and Recommendations}

The results obtained in this study indicates that although a large number of HCE workers sampled have knowledge of bio safety especially in Abuja, showed little understanding and practice of bio safety. It is recommended that HCEs and governments should expose their personnel to training and retraining. The use of improper waste disposal methods was recorded in Abuja during this study. It is recommended that waste disposal regulations should be enacted and that appropriate arm(s) of government in the Federal Capital Territory, Abuja must be committed to enforce the regulations as is done in Lagos.

\section{References}

1. Zaid A, Hughes HG, Poceddu E, Nicholas E (2001) Glossary of biotechnology for Food and Agriculture: A Revised and Augmented Edition of the Glossary of Biotechnology and Genetic Engineering. Publishing and Multimedia Service. Food and Agricultural Organization (FAO) Rome.

2. Centres for Disease Control and Prevention (CDC) (2010) Biosafety in Microbiological and Biomedical Laboratories. US Department of Health and Human Services. National Institutes of Health F $5^{\text {th }}(E d n$,$) pp. 415$.

3. World Health Organization (2000) Hazardous Wastes from Health Care Facilities. WHO, Rome, sItaly.

4. Stephen OA, Elijah IO (2011) Healthcare waste management in Nigeria: A case study. Journal of Health and Epidemiology 3(3): 99-110.

5. Garcia LS (2007) Diagnostic Medical Parasitology (17 ${ }^{\text {th }}$ edn.), American Society Microbiology. Washington pp. 1026.

6. Ohakwe O Johnson (2011) A Statistical Analysis of Medical Waste Generation, Management and the Health Implications: A Case Study of Enugu Metropolis, Enugu State, Nigeria. Continental J Applied Sciences 6(1): 25-30.

7. Santamaria J, Toranzos GA (2003) Enteric pathogens and soil: A review. Intestinal Microbiology 6(1): 5-9.

8. Mordi RM, Ngwodo POA (2007) A study of blood and gastro-intestinal parasites in Edo State. African Journal of Biotechnology 6(19): 22012207.

9. Cabrera HA, Porter J (1958) Survival time and critical temperatures of various strains of Entamoeba histolytica. Experimental Parasitology 7(3): 285-291.

10. Bingham AK, Jarolli EL, Meyer EA (1979) Gardia sp: Physical factors of excystation in vitro; and excystation versus eosin exclusion as determinants of viability. Experimental Parasitology 47(2): 284-291.

11. Pecson BM, Nelson KL (2005) Inactivation of Ascaris suum eggs by Ammonia. Environmental Science and Technology 39(20): 7909-7914.

12. Fayer R, Graczyk TK, Lewis EJ, Trout JM, Farley CA (1998) Survival of infectious Cryptosporidium parvum oocysts in seawater and eastern oysters (Crassostrea virginica) in the Cheaspeake Bay. Applied Environmental Microbiology 64(3): 1070-1074. 
(c) (P) This work is licensed under Creative

Submission Link: https://biomedres.us/submit-manuscript.php

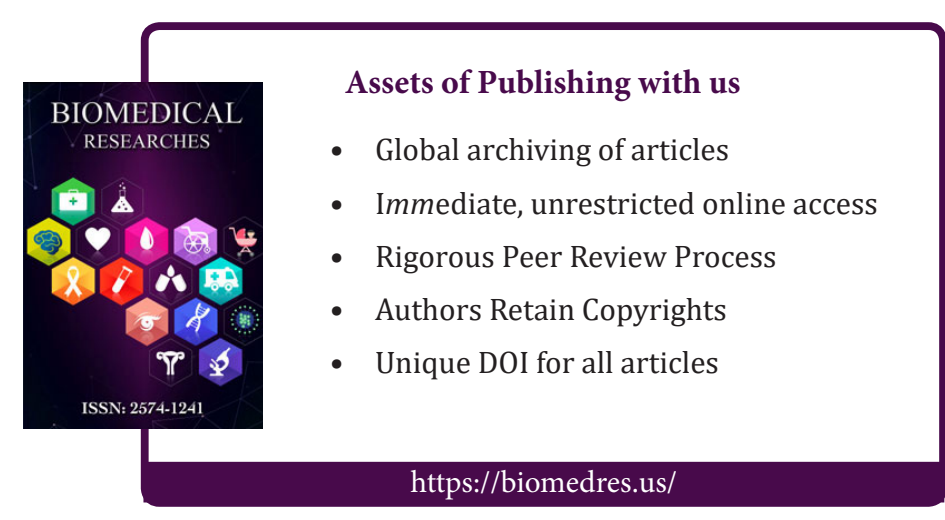

